\title{
Multi-Element Fiber Technology for High-Capacity Optical Communication Systems
}

\author{
V. J. F. Rancaño, S. Jain, T. C. May-Smith, J. K. Sahu, P. Petropoulos and D. J. Richardson \\ Optoelectronic Research Centre, University of Southampton, Southampton, UK SO17 IBJ \\ vjfr1u10@orc.soton.ac.uk
}

\begin{abstract}
Multi-element fiber technology is presented as an alternative approach to high capacity communication systems based on spatial division multiplexing. This talk discusses the advantages and potential drawbacks of this technology with respect to multicore and multimode approaches. OCIS codes: (060.2310) fiber optics; (060.2330) fiber optic communications
\end{abstract}

\section{Introduction}

The evolution of optical wavelength division multiplexed (WDM) systems towards more cost- and power-effective solutions is a necessity in order to cope with the expected growth of data traffic in the upcoming years [1]. Since the initial development of WDM in the mid-nineties, several approaches have been proposed to increase the transmission capacity of such systems, including the use of polarization division multiplexing and advanced modulation formats supported by the use of high speed digital signal processing and flexi-grid channel allocation). Use of either $\mathrm{C}+\mathrm{L}$ split-band amplification, multi-pump Raman amplification may provide scope for further (albeit somewhat limited) bandwidth extension, however to date such technology has not been considered sufficiently costeffective for commercial deployment. New solutions, both from a capacity and economic perspective are thus now required.

Space division multiplexing (SDM), by which parallel elements in WDM systems can be grouped to offer economic benefits, has emerged as one solution and has attracted considerable interest in recent years. The two main implementations of SDM rely on either multi-core fibers (MCF) [2] or few/multi-mode fibers (MMF) [3]. When the crosstalk levels between the spatial channels of an SDM system are above $25 \mathrm{~dB}$, these approaches do not provide similar levels of performance and reliability as conventional WDM unless electrical multiple-input-multiple-output (MIMO) processing is used to accommodate the spatial channel interference [4]. However, MIMO processing decreases the cost efficiency of SDM solution, requiring one additional component per wavelength and significant additional electrical power. In addition, for correct crosstalk correction, all the spatial channels must be accessed simultaneously, which limits network flexibility. The maximum attainable number of spatial channels using MCF is limited by the decreasing robustness of the fiber as its outer diameter grows; to date, as many as 19 independent cores have been demonstrated, however this already appears to be close the achievable limit of what can be practically achieved [2]. No such restrictions exist for MMF however the attenuation of the modes grows exponentially with the order of the mode [5], and since all supported modes must be used for MIMO to work properly, the effective range of MMF systems is that of the most lossy mode, leading to the need for more frequent electrical regeneration as the number of modes grows. Moreover, the complexity of the MIMO processing also increases rapidly as the number of modes is increased.

Multi-element Fibers (MEF) have been proposed as an alternative to these existing approaches [6-8]. This fiber geometry exhibits very low levels of crosstalk, avoiding the requirement for MIMO processing, is fully compatible with standard single mode fiber (SSMF) systems and offers several potential benefits from a device integration perspective, making it a suitable candidate for an effective implementation of SDM in commercial optical networks.

\section{Multi-Element Fibers}

An MEF consists of a bundle of single-mode fiber-elements drawn together in a common polymer coating, each fiber-element has an independent core and cladding, as shown in Fig. 1. This geometry allows each of the fiberelements to be smaller in diameter than a SSMF since having the fiber-elements drawn within a common coating has the potential of reducing the micro-bending losses relative to individual fibers of the same size. Moreover, this also alleviates the maximum fiber diameter mechanical reliability constraint associated with MCF and should allow for significant scaling in element (core) number. The in-situ bundling process of MEF fabrication eliminates the need for a two-stage fabrication process that is required for ex-situ bundling of SSMF, and has the advantage of being more compact than an equivalent ex-situ fiber bundle (albeit not as compact as an MCF). 


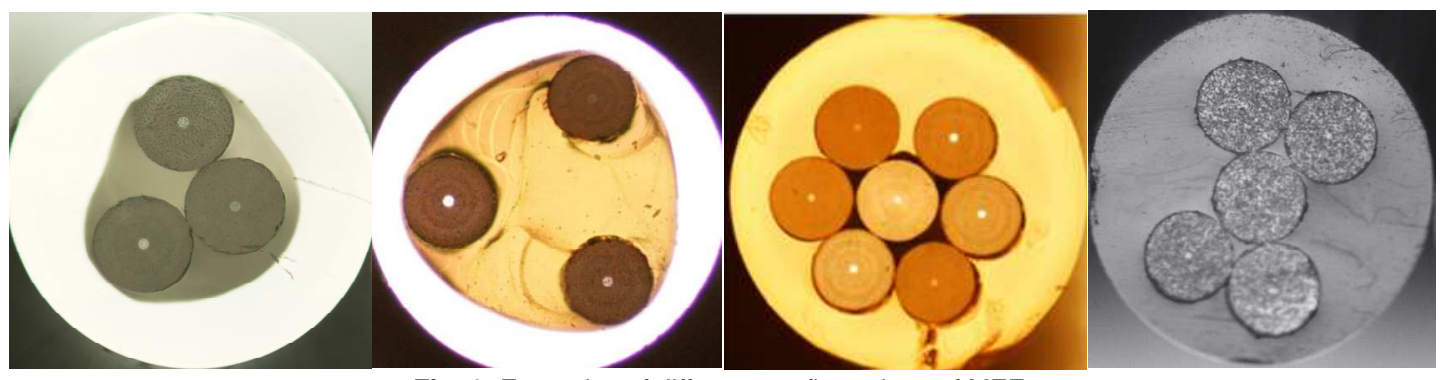

Fig. 1: Examples of different configurations of MEF.

The MEF geometry provides easy access to the individual fiber-elements simply by removing the common coating. In this manner, each fiber-element can be spliced individually to SSMF components using standard splicers and to provide crosstalk-free connections. Therefore, there is no need for any custom fan-in/fan-out devices to be developed.

The distance between the MEF fiber-elements must be carefully controlled during the manufacturing process in order to achieve the required degree of robustness and avoid the introduction of any additional losses. The spacing for a transmission fiber must be such that the fiber-elements are very close, but not physically in contact with one another, resulting in a compact implementation without performance degradation [6]. The attenuation and dispersion characteristics of MEF have been observed to be similar to those of SSMF drawn from the same starting preform [8], showing no additional penalty with respect to SSMF, although this remains to be proven at the $0.2 \mathrm{~dB} / \mathrm{km}$ level. MEFs fabricated so far exhibit crosstalk levels below $100 \mathrm{~dB}$ between the various fiber-elements [7].

An important feature of the MEF is that it lends itself directly to the implementation of SDM amplifiers. If the fiberelements are made to optically contact one another, a cladding-pumped multi-element erbium doped fiber amplifier (ME-EDFA) can be manufactured [8]. By making the central fiber-element a pump delivery fiber of pure silica with no core, pump light can be coupled into the ME-EDFA erbium-doped fiber-elements [7]. Since a single multimode pump diode is shared among all the fiber-elements this approach offers a cost effective SDM amplifier solution.

MEF-based systems do not require the use of MIMO processing because of the extremely low levels of crosstalk and the crosstalk-free splicing provides an inherent compatibility with SSMF. These combined features offer the potential to build cost-effective, scalable, backward compatible SDM systems.

In addition to the longer term application of MEF technology in SDM systems, the component sharing provided by the ME-EDFA can also be applied to improve the economics of existing SSMF-based networks. For example, in the implementation of a multi-channel amplifier, or as a more integrated $\mathrm{C}+\mathrm{L}$ split band amplifier device [9]. To this end we have recently demonstrated a flat gain and noise figure $\mathrm{C}+\mathrm{L}$ band amplifier pumped using just a single lowcost, multimode pump diode. In the same manner, if each of the fiber-elements of the ME-EDFA is doped with a different active element, a different kind of split-band amplifier could potentially be implemented with the same favorable economics deriving from pump sharing.

\section{Conclusions}

MEF technology exhibits features that make it a strong candidate for a flexible, crosstalk-free and robust SDM solution that can meet the requirements of commercial optical transmission systems. Additionally, the component sharing provided by this technology allows a more cost effective implementation of integrated multichannel and split-band amplifiers that can be applied to high-capacity SSMF-based optical communication systems.

[1] "Metro Network Traffic Growth: an Architecture Impact Study" Alcatel-Lucent Strategic White Paper. http://resources.alcatellucent.com/asset/171568.

[2] J. Sakaguchi et al., "Large-capacity transmission over a 19-core fiber" OFC OW1L3 (2013).

[3] V.A.J.M. Sleiffer et al., "73.7 Tb/s (96x3x256-Gb/s) mode-division-multiplexed DP-16QAM transmission with inline MM-EDFA" ECOC Th.3.C.4 (2012).

[4] P. Winzer et al., "Penalties from In-Band Crosstalk for Advanced Optical Modulation Formats" ECOC Tu.5.B.7 (2011).

[5] G. Yabre, "Comprehensive Theory of Dispersion in Graded-Index Optical Fibers" Journal of Lightwave Technology (18),2, 166 (2000).

[6] V. J. F. Rancaño et al, "First Demonstration of an Amplified Transmission Line Based on Multi-Element Fibre Technology" ECOC PD1.C.2 (2013).

[7] J. K. Sahu et al, "Multi-Element Fiber for Space-Division Multiplexing” Proc. SPIE 9009, Next-Generation Optical Communication: Components, Sub-Systems, and Systems III, 90090C (2013).

[8] S. Jain et al., "Multi-Element Fiber for Space-division Multiplexing Operations" Opt. Express, 22 (4), 3787-3796 (2014).

[9] S. Jain et al. "Cladding-pumped Er/Yb Multi-Element Fiber Amplifier for C+L band Operations" OFC M2J.3 (2014). 